\title{
CLASSIFICATION, EPIDEMIOLOGY AND AETIOLOGY OF ORAL RECURRENT APHTHOUS ULCERATION / STOMATITIS
}

Zain R.B. Classification, epidemiology and aetiology of oral recurrent aphthous ulceration / stomatitis, Annal Dent Univ Malaya 1999; 6: 34 - 37.

\section{INTRODUCTION}

The most prevalent oral mucosal lesions are aphthous ulcerations commonly referred to as "canker sores". The clinical characteristic of oral recurrent aphthous ulceration/stomatitis(ORAS) is well defined and can be partly described as an oval or rounded ulcer covered by a grey-white or yellowish fibrinous exudate and surrounded by an erythematous halo. There is intense or moderate pain and the ulcers heal in about $10-14$ days for the more common type and more than 2 weeks for the severe type.

Recurrence of the ulcers occurs at intervals within a year or over several years(1). Variations of ORAS described above have made studies on aetiology and treatment difficult to interpret due to differing descriptions of differing diseases with similar clinical signs and symptoms and possibly differing aetiologies. A classification that was considered useful as a working model for ORAS was formulated in 1978(2). While the classification of ORAS had been widely accepted since 1978, the cause for ORAS is still unknown and its aetiology in general remains unclear. However, its immunopathogenesis is now becoming more clearly defined as evidenced and described by many researchers(3).

\section{CLASSIFICATION}

The term aphthae was first used by Hippocrates(460$370 \mathrm{BC})$ in relation to disorders of the mouth. However, only in 1898 that the first clinical description of this term was documented by von Mikulicz and Kummel(4). They described 'Mikulicz aphthae' which is now synonymous with minor aphthous ulcers. In 1911, Sutton further described another type of recurrent ulcers termed 'periadenitis mucosa necrotica recurrens'(1). Herpetiform ulcer was described by Cooke in 1961 and is now regarded as one of the recurrent ulcers but separate from the aphthous ulcers. The name herpetiform ulcers denotes multiple small ulcers per attack mimicking a true herpetic lesion but is not caused by Herpes virus(1).

Many researchers described ORAS as being a disease of the oral mucosa characterized by recurrent, painful, single or multiple well-demarcated ulceration with peripheral red halo where healing takes place with or without scarring. These varied clinical presentations and the occurrence of some in mucocutaneous-ocular syndromes led to a classification proposed by Donatsky in 1973(5). Donatsky's classification was based on current research during that time period as listed below:

1. Stomatitis aphthosa recurrens, which is similar to Mikulicz's aphthae, described by Miculicz and Kummel in 1898.
Rosnah Binti Zain

Department of Oral Pathology, Oral Medicine \& Periodontology Faculty of Dentistry University of Malaya 50603 Kuala Lumpur Malaysia

2. Stomatitis aphthosa recurrens cicatricicans, which is similar to periadenitis mucosa necrotica recurrens, described by Sutton in 1911.

3. Stomatitis aphthosa recurrens herpetiformis, which is similar to herpetiform ulcers, described by Cooke in 1961.

4. Mucocutaneous-ocular syndromes with aphthous-like stomatitis

In 1978 a classification thought to be useful as a working model for recurrent oral ulcerative diseases in man was documented arising from a consensus from a number of researchers(2). Since then, this classification was accepted and further documented in 1980 by WHO(1). This classification is as presented below:

1. Minor aphthous ulcers

2. Major aphthous ulcers

3. Herpetiform ulcers

Except for the terminology used, the latter classification was similar to that described by Donatsky in 1973(5) where 'minor aphthous ulcer' is synonymous 'stomatitis aphthosa recurrens', 'major aphthous ulcer' is synonymous with 'stomatitis aphthosa recurrens cicatricicans' and 'herpetiform ulcer' is synonymous with 'stomatitis aphthosa recurrens herpetiformis'. Mucocutaneous-ocular syndromes with aphthous-like stomatitis as classified by Donatsky(5) were not included in this classification of 1978. Instead, a similar condition was recognized as its own entity called Behcet's syndrome that was classified separately into different subtypes as below:

1. Muco-cutaneous(oral and genital lesions with or without skin lesions)

2. Arthritis(oral, genital and skin lesions with arthritis)

3. Neuro-ocular(oral, genital and skin lesions and neurological symptoms)

\section{EPIDEMIOLOGY}

Different types of prevalence had been used for studies on ORAS by different investigators. In view of the natural history and recurrent nature of these lesions, crosssectional clinical surveys tend to underestimate the true prevalence of an ORAS, as active lesions may not be present at the time of the examination(6). Three types of 
prevalence have been used and reported in the literature for aphthous ulcers namely the prevalence for 'current uicers', for 'history of ulcers (throughout life)' and 'history of ulcers (within the last 2 years)' $(7,8)$. The prevalence for 'current ulcers' can be considered as average point prevalence (APP) where the presence of the ulcer/ulcers was detected at the time of the oral examination. The prevalence for 'history of ulcers (throughout life)' can be considered as self-reported lifetime prevalence (SRLP) where the subjects were asked whether they have ever had such ulcers. The prevalence for 'history of ulcers (within the last 2 years)'can be considered as a self-reported twoyear prevalence (SRTP) where the subjects were asked whether they ever had such ulcers within the last 2 years $(9)$. Variation in prevalence reported in different studies can partly be attributed to the usage of differing type of prevalence. For example, in Axéll's study consisting of 20,333 Swedish population above 15 years old, the APP of ORAS was found to be lower $(2 \%)$ than the SRTP $(17.7 \%)(8)$. Such result would be expected as the APP of clinically present ulcers represent only a small component of either SRTP or SRLP since the chances of capturing aphthous ulcers on a given day are small(6). For the purpose of discussing and comparing prevalence of ORAS in this paper, the terms APP, SRLP and SRTP will be used.

Samples selected for the study of ORAS may also be a contributing factor to the variation of prevalence of ORAS between studies. Most of the studies on ORAS prevalence have used samples of convenience such as health professionals/dental and medical students(7), schoolchildren(6), clinic/hospital-based patients(10-12), industrial workers(13) and army personnel(14). As the predisposing factors for ORAS includes systemic and genetic factors, the wide variations observed between these studies can also be attributed to differing samples selected(7).

Similarly, samples studied maybe age biased. Kleinmann et al had shown that the age-specific SRLP of ORAS ranged from about $21.7 \%$ at age 6 to $41.1 \%$ at age 17 in a study of 39,206 U.S. schoolchildren(6).

In general, the prevalence (mostly the minor type) of ORAS that has been reported in the literature varies from $0.4-66 \%(15,17)$ ). The lowest prevalence of $0.4 \%$ was a SRTP+APP and was reported by Taiyeb Ali et al in elderly $(>60$ years) Malaysian. The highest prevalence of $66 \%$ was a SRLP in a study by Ship et al (1967)(15) on dental and medical students.

A search of the literature showed that only 3 ORAS prevalence studies were conducted on the general population worldwide. One study was conducted in a random sample of Danish over 65 years of age where the APP of ORAS was 1\%. A study by Axéll in 20,333 Swedish county residents above 15 years of age showed the SRTP + APP of $19.7 \%(8)$. Fahmy found that the SRLP of ORAS was $18 \%$ in 9000 Kuwaiti Arabs, $35 \%$ in 11,000 Non-Kuwaiti Arabs and a low SRLP of $5.0 \%$ in Bedouins(16). Very recently Zain reported an APP of $0.5 \%$ in 11,697 randomly selected Malaysian population(9).
The SRLP of ORAS in professionals/professional school student groups varies from $43.1 \%$ in 10,531 Health Sciences students in 21 countries(7) to $66.2 \%$ in 343 medical/dental students in the United States(15). Two other studies from the United States on these population groups were on 1,788 Professional school students and 704 Health professionals where the SRLP were $55.0 \%$ and $50.7 \%$ respectively $(7,15)$.

The SRLP of ORAS in 1,738 medical and surgical patients of all ages in the United Kingdom was found to be $19.3 \%(10)$ which is lower than the SRLP in dental clinic outpatients. The SRLP of ORAS in 3,106 dental clinic outpatients from a Thai dental school was found to be $46.7 \%(12)$ while the SRTP+APP of $48.3 \%$ was found in 234 dental clinic outpatients in a similar study in another Thai dental school(11).

In Malaysia, 2 studies were conducted on dental clinic outpatients. One was on 233 dental clinic outpatients from a dental school where the SRTP +APP was $27.0 \%(11)$ while the other was on 999 dental clinic outpatients from a government dental clinic where the SRTP + APP was $28.0 \%(18)$. Among factory workers in Malaysia, the SRTP+APP was found to be $38.9 \%$ for glass factory workers and $56.5 \%$ for steelmill workers(13). Another study in Malaysia was conducted on 1013 army personnel (all men) where the SRTP+APP for ORAS was $19.9 \%(14)$. This value seemed lower than most of the other Malaysian studies and maybe due to the fact that all the subjects were men. Some studies have shown that the prevalence of ORAS was more frequent in women than men(19).

The prevalence of ORAS also varied tremendously depending on the age range of the population selected. Axéll's study on the Swedish population had shown a higher prevalence of $26.7 \%$ in the $15-24$ age group as compared to $7.5 \%$ in the $65-74$-age group(8). Similar age-specific prevalence was also reported in US schoolchildren(6) where they observed the SRLP ranging from $21.7 \%$ at age 6 years to $41.1 \%$ at age 17 years. Ferguson et al had also shown such differences in the prevalence of 25-30 year old women $(25.4 \%)$ and 45-56 year old women $(6.1 \%)(19)$.

In summary, the prevalence of ORAS is dependent on whether it is that of current ulcers or selfreported life or 2-years prevalence. Age and gender distribution of the subjects and type of population studied can also influence the prevalence of ORAS.

\section{AETIOLOGY}

The actiology of ORAS is unknown and remains unclear. Other than the presence of ulcers and pain experienced for some patients, most of these patients are generally well. Many studies have shown that ORAS has many predisposing factors. In about $10-20 \%$ of patients, deficiencies of iron, folic acid or vitamin B12 was reported. About $2-3 \%$ of patients with ORAS were related to coeliac disease (gluten-sensitive enteropathy)(20). Haematinic deficiencies seemed to be secondary to coeliac disease. Other underlying systemic diseases and immunodeficiencies presenting with aphthous-like oral ulcers are agranulosytosis, neutropenias (cyclic and 
neutropenia from other causes such as autoimmune neutropenia) and HIV-seropositive patients. The association of Behcet's Syndrome and its variants with recurrent aphthous stomatitis have long been recognized and documented. Oral ulcerations similar to ORAS may also be associated with other syndromes such as Sweet's syndrome (acute febrile neutrophilic dermatosis-raised erythematous plaques on face, neck, chest and extremities accompanied by fever and general malaise), MAGIC syndrome/FAPA Syndrome (fever, aphthous, pharyngitis and cervical adenitis)(20-21) In a small group of women with ORAS, cyclical oral ulceration occurred in relation to the luteal phase of the menstrual cycle and presumedly modulated by changing levels of progesterone since the occurrence of ORAS in these cases appears unrelated to psychologic factors $(19,20)$. Many researchers have indicated stress as one of the predisposing factor for ORAS in some susceptible people. However, there are insufficient data supporting this(21).

Food allergy (hypersensitivity reactions to exogenous antigens) has been suggested as having a potential role in causing ORAS(20). However, at a recent meeting Report of the third European Congress of Oral Medicine, it was summarized that hypersensitivity reaction to exogenous antigen other than gluten does not have a significant role in ORAS(21). There has also been evidence of a familial basis in some of these susceptible individuals where patients with a family history may develop ORAS at an earlier age and with more severe symptoms than those without a family history. There is also a high correlation of ORAS in identical twins as compared to non-identical twins(20). However, studies of patients of different ethnic groups have failed to show significant associations between a serologically determined HLA antigen and susceptibility of ORAS, thus making the immunogenetic basis questionable. There is also now evidence that drugs such as the non-steroidal anti-inflammatories can also give rise to aphthous-like ulcers. ORAS may also be initiated by local factors such as trauma in susceptible person. In areas of mucosal keratinization, ORAS is uncommon(21). This further explained reports that the prevalence of ORAS is lower in smokers(11) since smoking causes keratinization of the oral mucosa.

Eventhough there are reports of immunological changes in ORAS, evidence for ORAS as an autoimmune disease is so far lacking or unreliable(21). There appears to be a genetically determined immunological reactivity to unidentified antigens. The latter is most probably microbial and such aetiology has long been suggested while the search for a viral cause has been and is still ongoing(3). Eventhough Varicella-zoster virus and Cytomegalovirus IgM antibody titers are increased during ulcer recurrences, intralesional viral antigens and DNA have not been identified. It has been suggested based on past and current evidence that ORAS may be due to changes in cell-mediated immune responses and crossreactivity with Streptococcus sanguis (S. sanguis) or a heat shock protein. The L-form of $\mathrm{S}$. sanguis was initially isolated from ORAS patients and further analysis showed this to be a strain of S. mitis. A cross-reactivity between a streptococcal $60-65 \mathrm{kDA}$ heat shock protein and the oral mucosa has been demonstrated as well as significantiy raised levels of antibodies to heat shock protein in ORAS patients. The lymphocytes in ORAS patients also have reactivity to a heat shock protein peptide. Cross-reactivity between the $65 \mathrm{kDa}$ heat shock protein and the $60 \mathrm{kDa}$ human mitochondrial heat shock protein lead to a $T$ cellmediated response to antigens of $\mathrm{S}$. sanguis that cross react with mitochondrial heat shock protein and inducing oral mucosal damage(21).

The immunopathogenesis of ORAS seemed more clearly defined. A variety of adhesion molecules are expressed in ORAS and probably control the movement of leukocytes up into the epithelial layer where leukocytemediated keratinocyte lysis occurs(3). Cytotoxic $\mathrm{T}$ - cells respond to oral keratinocyte-associated antigen (a target antigen residing within the epithelial compartment). Once on-site, a variety of lymphokines including tumour necrosis factor (thought to play a role in the cytolytic process) are released. As the lesion progress to ulcers, there is an increase in local cytotoxic cell population and (presumedly cytokine related) increased expression of HLA class 1 and II antigen on epithelial cells. Current evidence suggested that immunologically mediated cytotoxicity of the oral epithelium is important in the development of ulcers in ORAS(21).

\section{REFERENCES}

1. World Health Organisation. Guide to epidemiology and diagnosis of oral mucosal diseases and conditions. Community Dent Oral Epidemiol 1980; 8: 1-26.

2. Hook JJ, BenEzra D, Cohen L, Dattner A, DetrikHooks B, Lehner T, Mebus C and Openshaw H. Classification, pathogenesis and etiology of recurrent oral ulcerative disease and Behcet's syndrome. I Oral Pathol 1978; 7: 436-438.

3. Eversole LR. Immunopathology of oral mucosal ulcerative, desquamative, and bullous diseases. Selective review of the literature. Oral Surg Oral Med Oral Pathol 1994; 77: 555-571.

4. Ship JA. Recurrent aphthous stomatitis. An update. Oral Surg Oral Med Oral Pathol Oral Radiol Endod 1996; 81: 141-147.

5. Donatsky O. Epidemiologic study on recurrent aphthous ulcers among 512 Danish Dental students. Community Dent Oral Epidemiol 1973; 1: 37-40.

6. Kleinman DV, Swango PA, Pindborg JJ. Epidemiology of oral mucosal lesions in United States school children: 1986-87. Community Dent Oral Epidemiol 1994; 22: 243-253.

7. Kleinman DV, Swango PA, Niessen LC. Epidemiologic studies of oral mcosal conditions methodologic issues. Community Dent Oral Epidemiol 1991; 19: 129-140.

8. Axéll $\mathrm{T}$, Henricsson V. The occurrence of recurrent aphthous ulcers in an adult Swedish population. Acta Odontologica Scand 1985; 43: 121-125.

9. Zain RB. Prevalence of recurrent aphthous in Malaysia - an epidemiological update. J Oral Sci -in press. 
10. Sircus W, Church R, Kelleger J. Recurrent ahthous ulceration of the mouth. A study of natural history, etiology and treatment. Q J Med 1957; 28: 235-249.

11. Axéll T, Zain RB, Siwamogstham P, Tantiniran D, Thampipit J. Prevalence of oral soft tissue lesions in outpatients at two Malaysian and Thai Dental Schools. Community Dent Oral Epidemiol 1991; 18: 95-99.

12. Pongissawaranun W, Laohapand P. Epidemiologic study on recurrent aphthous stomatitis in a Thai dental patient population. Community Dent Oral Epidemiol 1991; 19: 52-53.

13. Zain RB, Abdullah F, Jalaluddin M. The oral health of glass factory workers - a comparative study. Dent J Malaysia 1995; 16: $44-48$.

14. Zain RB. A preliminary report on the prevalence of oral mucosal lesions in army personnel in Johor, Malaysia. Dent J Malaysia 1995; 16: 37-39.

15. Ship II, Brightman VJ, Laster LL. The patient with recurrent aphthous ulcerations and the patient with recurrent herpes labialis: A study of two population samples. J Am Dent Assoc 1967; 74 : 645-653.

16. Fahmy MS. Recurrent aphthous ulcerations in a mixed Arab community. Community Dent Oral Epidemiol 1976; 4: 160-164

17. Taiyeb Ali, T.B., Razak, I.A., Raja Latifah, R.J. and Zain, R.B.: Oral mucosal Lesions in elderly Malaysians. Gerodontology, 12: 37-40, 1995

18. Zain RB, Axéll T. Prevalence of oral mucosal lesions in 999 outpatients at a government Dental Clinic in Malaysia. Dent J Malaysia 1995; 15: 9-11.

19. Ferguson MM, Carter J, Boyle P. An epidemiological study of factors associated with recurrent aphthae in women. J Oral Med 1984; 39: 212-217.

20. Porter, S.R., Scully, C. and Pedersen, A.: Recurrent aphthous stomatitis. Crit. Rev. Oral Biol. Med., 9: 306 - 321, 1998

21. Meeting Report. Third European Congress of Oral Medicine. Oral diseases 1997; 3: 43-48. 